\title{
Acquired lymphangioma circumscriptum of the vulva treated with ablative radiofrequency
}

\begin{abstract}
(LC) is a benign disorder involving the lymphatic system of skin and subcutaneous tissue. A case of acquired LC of the vulva in a57-year-old woman with a 10 -15 year history of lymphoedema of the left leg and vulvar lesions (vesicles and papulovesicles) following cervical cancer operation and subsequent radio therapy is presented. The patient was treated with ablative radiofrequency and was free of lesions for 6 months.
\end{abstract}

Keywords: lymphangioma circumscriptum, dermoscopy, ablative radiofrequency, radical vulvectomy
Volume 5 Issue 5 - 202I

\author{
Jelica PVukićević,' Tijana P Pejić \\ 'Medical faculty, University of Belgrade, Clinical center of \\ Serbia,Serbia \\ ${ }^{2}$ Clinic of Gynecology and Obstetrics, Clinical Centre of Serbia, \\ Serbia
}

Correspondence: Jelica PVukićević, Medical faculty University of Belgrade, Clinical center of Serbia, Serbia, Tel +38I I I3662452, Email jvukicevicsretenovic@gmail.com

Received: September 30, 2021 | Published: October 15, 2021

\section{Introduction}

Lymphangioma circumscriptum (LC) is a rare benign cutaneous disorder involving lymphatic channels of skin, subcutaneous tissue and sometimes muscle. It was initially described by Fox and Fox in 1878. The term LC was coined by Malcom and Morris in $1889 .{ }^{1}$ Vulvar $\mathrm{LC}$ is often an acquired form of the disease which arises secondary to surgery/ radiotherapy for carcinoma of the cervix or vulva, tubercular inguinal lymphadenitis and Crohn's disease of the vulva. ${ }^{1}$ Among 44 reported cases (in any language) of vulvar LC by December 2014, only 24 cases were acquired LC. ${ }^{4}$ It is not a life threatening disease, but may cause serious impairment of a patients quality of life. Radio frequency is described as one of the treatment options in some other, non-vulvar localizations of LC. . $^{2,3}$

Herein is presented a rare case of secondary vulvar LC in a57year- old woman treated with ablative radiofrequency.

\section{Case report}

A 57-year-old female patient was referred from the Clinic of Gynecology, Clinical Center of Belgrade, Serbia, Faculty od Medicine Belgrade, with complaints of left lower-limb lymphedema, oozing lesions on the labia majus on the same side, and history of cervical cancer surgery 13 years ago. Lymphoedema of the left leg appeared 7 years after surgery, and changes on the labia after 10 years. According to the medical documentation, radical hysterectomy and adjuvant radiation therapy were performed under the diagnosis of a stage $1 \mathrm{~B}$ squamous cell carcinoma of the cervix.

On examination, lymphoedema of the left leg was noticed. On the left labia majus, lymphoedema with scattered vesicles and keratotic papulovesicles were present (Figure 1a).Oozing and drainage of clear fluid was seen from vesicles. Dermoscopy of the lesions showed multiple white-yellowish well-circumscribed roundish areas (lacunae) surrounded by pale septa (Figure 1b). Biopsy of a vesicle was taken. Histopathology revealed focal acanthosis in the epidermis and dilated lymphatic vessels filled with eosinophilic contents in the papillary dermis (Figure 1c). Immuno histochemistry showed positive reaction for CD31 and D2-40 (Figure 2a,b) in the endothelial cells of the lymphatic vessels, and negative reaction for HHV8.

The therapy with ablative radiofrequency was applied. A radiofrequency generator- Ellmann Surgitron, model FFPF.EMC, New York, USA was used. Lesions were excised with a loop electrode with the diameter of $10 \mathrm{~mm}$ in a cut mode (Figure 2c). Hemostasis was achieved with a ball electrode measuring $2 \mathrm{~mm}$ operating in a coagulation mode. The whole procedure was done in short-term general anesthesia. The patient was given systemic antibiotic therapy with the wound dressing with sterile paraffin pad. After six months, a few new vesicles were noted and further gradual deterioration of the disease occured with oozing, infection and pain.
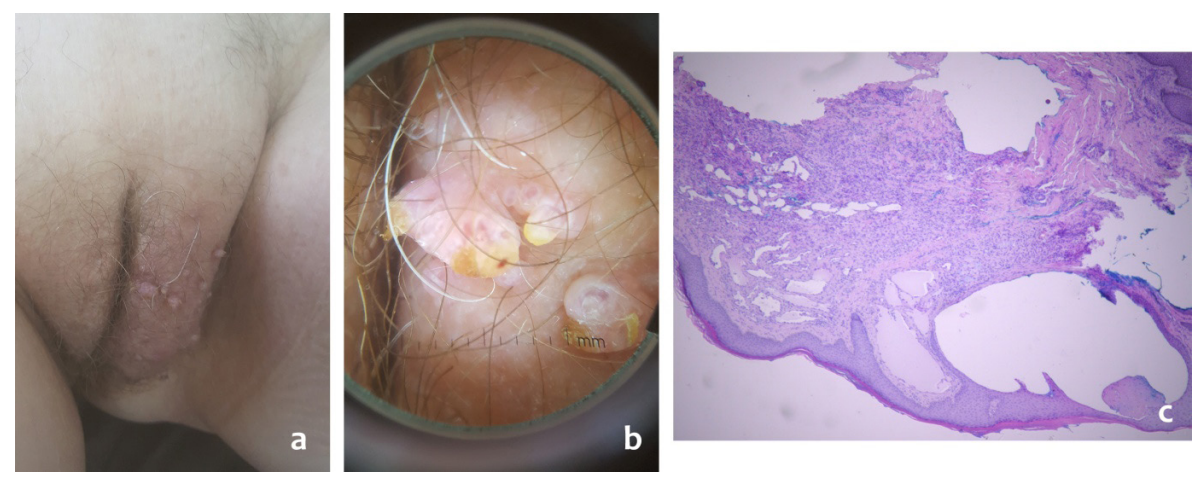

Figure I LC: clinical presentation (a), dermoscopic (polarised xI0) (b) and histopathological features (hematoxylin eosin xI00) (c). 

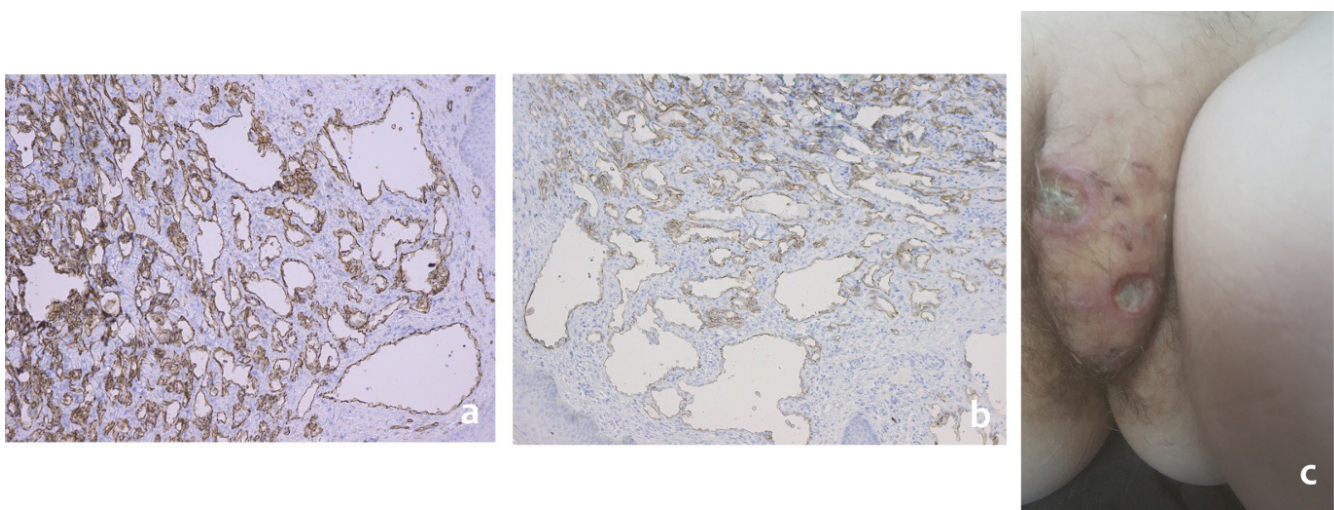

Figure 2 LC: $C D 3$ I and C2-40 postivity $(x \mid 00)(a, b)$; LC after treatment with ablative radiofrequency $(c)$.

\section{Discussion}

Lymphangioma circumscriptum (LC) is an uncommon disorder of lymphatic origin, which is divided into congenital or primary LC, which appears in early childhood due to inadequate development of lymphatic channels with no impairment of the main lymphatic drainage, and acquired LC, which occurs in adults. Vulvar LC is the most common entity among acquired LC and usually appears after radical hysterectomy with or without adjuvant radiation therapy for cervical cancer due to disruption of previously normal lymphatic system. According to the results of Gun Yoon and coworkers, 87,5\% of 24 cases with secondary vulvar LC were associated with cervical cancer treatment. The remaining three patients had Crohn's disease, reccurent cellulitis and leg edema. ${ }^{4}$

Other infectious diseases (filariasis, genital tuberculosis, sexually transmitted diseases), surgical trauma, keloids, scleroderma, rhabdomyosarcoma, may also be causes of vulvar LC.

Lesions typically arise 7-15 years after lymph node dissection and follow radiotherapy in females aged 22-75 years with mean age 48.5 years. ${ }^{1}$ However, Ikeda M., at all presented a56- year-old woman who noticed vulvar lesions 24 years after radical hysterectomy.

Clinically, LC is characterized by vulvar swelling, oozing, pruritus, pain. The patient feels discomfort and patients' quality of life and social contacts are affected. LC should be differentiated from condyloma acuminatum, herpes zoster, hydradenitis, molluscum contagiosum and fungating squamous cell carcinoma. Histopatholgically, these lesions are composed of multiple, superficial lymphatic vessels lined by endothelial cells with no atypical vascular lesions. Endothelial cells of the lymphatic vessels are positive for CD31, CD34 and D240, which is helpful for the confirmation of LC. ${ }^{5}$ HHV 8 was negative by immunohistochemistry. Dermoscopy of LC is a characteristic and useful additional diagnostic criterium, but histopathological verification is gold diagnostic standard.

There are several treatment modalities for LC that can be generally divided into radical surgery and other, palliative or conservative modalities such as radiotherapy, sclerotherapy, $\mathrm{CO} 2$ laser therapy. ${ }^{6,7}$

These conservative therapeutic options in vulvar LC are most commonly used in unilateral vulvar affections withfewer lesions on the vulva, less pronounced hyperkeratosis.

Data on the treatment of LC with ablative radiofrequency are not numerous. According to our best knowledge, data of the treatment of acquired vulvar LC with ablative radiofrequency are not available in English literature.
Omprakash HM and Rajendar SC treated a 29-year-old patient with congenital LC on the trunk with coagulation using radiofrequency current and there was no recurrence after 6 months. On the other hand, the same authors treated in the same way a 25 -year-old man with scrotal LC when relapses reported shortly after treatment. ${ }^{3}$ Sachdeva $\mathrm{S}$ treated a 16-year-old man with congenital LC on his left arm with radiofreqency ablation and no recurrence occurred after a year. ${ }^{2}$ In our patient, relapse occurred after 6 months. Perhaps, relapses after treatment of LC with ablative radio frequency can be explained by depth of penetration of radio waves which is equal to half the size of $2 \mathrm{~mm}$ ball electrode and reaches papillary and partially reticular dermal lymphatics. Puri $\mathrm{N}$ in his comments emphasizes that energy of the waves must penetrate into the dermis and subcutaneous tissue. Otherwise, the patient will only achieve short term palliative symptomatic relief and will have lesion recurrence. ${ }^{8}$ Similar ablative depth, $1.3 \mathrm{~mm}$, reaching $\mathrm{CO} 2$ laser.

Yoon G. et al, anlized eight patients to asses the clinical outcome of surgical treatment of acquired vulvar LC. All patients received radical abdominal hysterectomy with bilateral pelvic lymph node dissection due to cervical cancer with FIGO stage IIA in four, IB in three and IA in one patient. They also received radiation therapy, or concurrent chemoradiation therapy. Six of eight patients develop no recurrence. Authors propose that surgical treatment could provide a more long lasting answer compared to other treatment modalities. ${ }^{4}$ The same authors concluded that radicality of surgical primary tumor treatment seems to have more impact on the development of vulvar LC than the tumor stage.

Valente $\mathrm{K}$ et al also described a case of acquired vulvar $\mathrm{LC}$ treated by surgery with complete excision of the affected lymphatic channels, but they followed the patient for only 5 months. ${ }^{7}$ The choice of therapy depends on the number and intensity (presence of hyperkeratosis) of the lesions on the affected area. A smaller number of the lesions amd unilateral vular affection determined us to choose a non-surgical method.

Here is presented a rare case of vulvar LC treated with ablative radiofrequency. It should be emphasized that there is no official recommendation for LC therapy and that, in addition to all the above mentioned therapeutic modalities, LC remains a therapeutic challenge.

\section{Conflicts of interest}

The authors declare no conflict of interest.

\section{Acknowledgments}

None. 


\section{Funding}

None.

\section{References}

1. Ramesh MB, Cella SS, Srinath M, et al. Cutaneous lymphangiectasia of the vulva secondary to tuberculosis. Indian J Sex Transm Dis AIDS. 2012; 33(1):35-37.

2. Sachdeva S. Lymphangio macircumscriptum treated with radiofequency ablation. Indian J Dermatol. 2011;56:77-78.

3. Cmprakash HM, Rajendran SC. Lymphangioma circumscriptum (Mycrocystic lymphatic malformation): palliative coagulatin using radiofrequency current. $J$ Cut Aesthetsurg. 2008;1(2):85-88.

4. Yoon G, Kim HS, Lee YY, et al. Clinical outcomes of primary surgical treatment for acquired vulvar lymphangioma circumscriptum. Arch Gynecol Obstet. 2016 ;293(1):157-162
5. Akhavan S, Agah J, Nili F. Congenital lymphangioma circumscriptum of vulva presenting as multiple giant mass lesions: a case reprt and literature review. J Obstret Gynaecol Res. 2018;44(5):978-982.

6. Ikeda M, Muramatsu T, Shida M, et al. Surgical management of vulvar lyphangioma circumscriptum: tw case reprts . Tokai J ExpClin Med. 2011;36(1):17-20.

7. Valente K, Montgomery $\mathrm{K}$, Schultenover S, et al. Acquired vulvar lymphangioma circumscriptum after cervical cancer treatment: Case report. Gynecologic Oncology Reports. 2016;16:31-33.

8. Puri N. Treatment options of lymphangioma circumscriptum. Indian Dermatol Online J. 2015;6(4):293-294 